\title{
Differential microRNA expression profiling of mesothelioma and expression analysis of miR-1 and miR-214 in mesothelioma
}

\author{
VISHWA JEET AMATYA $^{1 *}$, AMANY SAYED MAWAS ${ }^{1,2^{*}}$, KEI KUSHITANI $^{1}$, \\ MOUCHIRA M. MOHI EL-DIN ${ }^{2}$ and YUKIO TAKESHIMA ${ }^{1}$
}

${ }^{1}$ Department of Pathology, Hiroshima University Graduate School of Biomedical and Health Sciences, Hiroshima, Japan;
${ }^{2}$ Department of Pathology and Clinical Pathology, Faculty of Veterinary Medicine, South Valley University, Qena, Egypt

Received November 2, 2015; Accepted December 11, 2015

DOI: $10.3892 /$ ijo.2016.3358

\begin{abstract}
Malignant mesothelioma is a highly aggressive cancer with poor prognosis and refractory to currently available therapies. Most of the patients with advanced invasive nature are not amenable to surgical resection and/or available anticancer therapy, thus development of novel effective therapeutic regimes is needed. Aberrant expression of microRNAs (miRNAs) has been proposed to contribute to carcinogenesis and aggressiveness of mesothelioma. We analyzed miRNA expression in mesothelioma cell lines using TaqMan miRNA expression array and found significant number of miRNAs, which showed increased or lost expression. We validated the increased expression of miR-182, and miR-183 in mesothelioma cell lines by individual miRNA assays and SmartFlare miRNA probes. We further investigated the miR-1, and miR-214, which were not expressed in mesothelioma cells by real-time RT-PCR. Transfection of mesothelioma cells, ACC-Meso-1 and CRL5915, with miRNA mimic (hsa-miR-1 mimic and hsa-miR-214 mimic) led to inhibition of cell growth, invasion and migration. We paid attention to PIM1, the target gene of both miR-1 and miR-214 miRNAs and which was found overexpressed in mesothelioma cells, and miR-1 and miR-214 mimic transfection of mesothelioma cell lines showed downregulation of PIM1 by western blot analysis. The miRNAs, miR-1 and miR-214, may play a role in carcinogenesis of mesothelioma thus might be considered as candidate therapeutic targets in mesothelioma.
\end{abstract}

\section{Introduction}

Mesothelioma, primarily associated with exposure to asbestos, causes 2,500 deaths per year in the United States alone (1).

Correspondence to: Professor Yukio Takeshima, Department of Pathology, Hiroshima University Graduate School of Biomedical and Health Sciences, 1-2-3 Kasumi, Minami-ku, Hiroshima 734-8551, Japan

E-mail: ykotake@hiroshima-u.ac.jp

${ }^{*}$ Contributed equally

Key words: mesothelioma, microRNA, miR-1, miR-214, PIM1, functional assay
There is a recent trend of decreasing incidence of mesothelioma in the USA and Canada (2), but its incidence is still rising in Europe, Japan (3) and in developing countries. Vital Statistics Data published yearly by Ministry of Health, Labor and Welfare, Japan indicated that there is an increasing trend in the death due to mesothelioma, from 500 cases in 1995 to 710 in 2000, 911 in 2005, 1109 in 2010 and 1,376 in 2014. It is a highly aggressive cancer with poor prognosis (4) and refractory to currently available therapeutic regimes $(5,6)$. In population-based studies, survival times ranged from 5 to 13.2 months (7). Although surgery is an option for patients with early stage mesothelioma, most patients present with advanced invasive stage are not amenable to surgical resection and development of new therapeutic regimes is needed.

MicroRNAs (miRNAs) are regulators of developmental and cellular pathways and most commonly silence the genes by binding to the 3'-untranslated region of target mRNA. This binding induces either mRNA degradation or inhibition of translation (8). More than $50 \%$ of miRNA genes are found in cancer-associated genomic regions and their altered expression in numerous cancers supports the notion that these small RNAs serve as a novel class of oncogenes or tumor suppressors (9). Recently, Truini et al reviewed the aberrant expression of miRNAs in mesothelioma for their role in carcinogenesis, diagnosis and prognosis (10). miRNAs such as miR-31 (11), $\mathrm{miR}-34 \mathrm{~b} / \mathrm{c}$ (12) as potential therapeutic target, miR-29c* (13) as prognostic biomarker, the miR-200 family (14) as diagnostic marker, miR-126 (15), miR-625-3p (16) as biomarker for early detection have been investigated.

We analyzed the miRNA expression in mesothelioma cell lines and found significant number of differentially expressed miRNAs between mesothelioma cell lines and non-neoplastic cells by TaqMan Low Density miRNA expression PCR array profiling. We focused on two highly expressed miRNAs, the miR-182 and miR-183, and two negligibly expressed miRNAs, miR-1 and miR-214, in mesothelioma cell lines and analyzed their functional role in mesothelioma cell lines.

\section{Materials and methods}

Mesothelioma cell lines and pleural tissue. Seven mesothelioma cell lines were included in the study. Five cell lines (CRL-2081, -5820, -5826, -5915, -5946) were purchased from 
the American Type Culture Collection (ATCC) (Manassas, VA, USA) and two cell lines (ACC-Meso-1, ACC-Meso-4) (17) from the RIKEN BioResearch Center (Tsukuba, Ibaraki, Japan). All cell lines were maintained in Roswell Park Memorial Institute 1640 medium with GlutaMAX (RPMI-1640) with 1\% kanamycin, $1 \%$ fungizone and $10 \%$ fetal bovine serum (all purchased from Gibco/Life Technologies, Tokyo, Japan). Non-neoplastic mesothelial cells were retrieved from the strip of pleura of patients who underwent autopsy for causes other than neoplastic lesions, and were snap-frozen immediately in liquid nitrogen and processed for total RNA extraction. The use of these samples conformed to the statement of Declaration of Helsinki Ethical principles for Medical Research.

RNA isolation. Total RNA containing small non-coding RNA was extracted from mesothelioma cell lines and non-neoplastic mesothelial cells using mirVana miRNA isolation kit (Ambion/ Life Technologies) according to the manufacturer's recommended protocol. The extracted RNA was directly quantified by the absorbance ratio at 260 or $280 \mathrm{nM}$ with a NanoVue Plus spectrophotometer (GE Healthcare BioSciences, Tokyo, Japan) and with fluorometer-based RNA Assay kit using Qubit 2.0 (Life Technologies). The integrity of total RNA was evaluated by capillary electrophoresis on RNA HighSens analysis kit using Experion automated electrophoresis system (Bio-Rad, Tokyo, Japan).

miRNA expression profiling. Total RNA $(1 \mu \mathrm{g})$ in $7.5 \mu$ l reaction volume was reverse-transcribed using Megaplex RT Primers, Human Pool set v3.0 using GeneAmp PCR systems 9700 (Applied Biosystems/Life Technologies) and $6 \mathrm{ml}$ of Megaplex RT product was used for miRNA expression profiling in TaqMan Low Density Array Human miRNA Cards v3.0 using 7900HT Fast Real-time PCR system (Applied Biosystems/Life Technologies) according to the manufacturer's instructions. The array card set consisted of 2 cards of 384 low density designed for analysis of 754 human miRNAs based in Sanger miRbase v14. Relative expression was determined using the $\Delta \Delta C t$ method and a $\geq 40 \mathrm{Ct}$ value was interpreted as amplification too low to quantify. miRNA expressions were analyzed after normalization to RNU6B (U6 snRNA, an endogenous control assay designed in both cards) using DataAssist software version 3.0 (Life Technologies) to calculate the fold change and hierarchical clustering of significant genes using JMP 9 software.

miRNA expression validation by TaqMan microRNA assays. Total RNA from mesothelioma cells ( $5 \mu$ l containing $10 \mathrm{ng}$ total RNA) was reverse transcribed with the TaqMan MicroRNA Reverse Transcription kit and $3 \mu \mathrm{l}$ of TaqMan MicroRNA assays specific for hsa-miR-182, hsa-miR-183, hsa-miR-1, hsa-miR-214 and endogenous controls (Life Technologies) using Eppendorf Mastercycler (Eppendorf AG, Hamburg, Germany). The resulting cDNA $(1.33 \mu \mathrm{l})$ was used as template in a total reaction volume of $20 \mu \mathrm{l}$ for real-time quantitative PCR analysis (qPCR) which was performed in triplicates with TaqMan microRNA assay primer/probes specific for hsa-miR-182, hsa-miR-183, hsa-miR-1, hsa-miR-214 and endogenous controls in TaqMan Universal PCR Master Mix
(Life Technologies) using 7900HT Fast Real-time PCR system (Applied Biosystems/Life Technologies).

Live cell miRNA validation by SmartFlare probes. SmartFlare technology detects RNA or miRNA in live cells. Mesothelioma cells were cultured in collagen-coated 24-well glass-bottom plates at a concentration of $1 \times 10^{4}$ cells per well, in $1 \mathrm{ml}$ of RPMI-1640 for $12 \mathrm{~h}$. SmartFlare probes (3 $\mu \mathrm{l})$ (Cy3 labeled miR-182-5p, miR-183-5p, and scramble control detection probes, purchased from Millipore) prediluted into $50 \mu \mathrm{l}$ of PBS were added to the each well in triplicates. Cells were incubated overnight $(\sim 16 \mathrm{~h})$ at $37^{\circ} \mathrm{C}$ and $5 \% \mathrm{CO}_{2}$ and analyzed with fluorescent microscope and digital picture was taken with similar light exposure for expression of miRNA.

Transient transfections of mesothelioma cells with miRNA mimics. All transfection studies were carried out in triplicates with $10 \mathrm{nM}$ of the each mirVana miRNA mimics by the Lipofectamine RNAiMax reagent (Lipofectamine) in OptiMEM media (Life Technologies) using collagen-plated 6-well plates for 2 or 3 days with exchange of miRNA mimics and Lipofectamine every $24 \mathrm{~h}$. Mesothelioma cell lines, ACC-Meso-1 and CRL 5915, were transfected with mirVana miRNA mimics specific for hsa-miR-1, hsa-miR-214, and negative control mimic (Life Technologies). After transient transfection with miRNA mimics, the cells were analyzed by various cell based assays.

Cell proliferation, cell cycle, migration and invasion assays. Cell proliferation assay was analyzed with Guava ViaCount reagent using Guava EasyCyte Mini Flow Cytometer (Guava Technologies/Millipore, Hayward, CA, USA). miRNA mimic transfected cells were incubated in RPMI-1640 media with $10 \%$ FBS in collagen-coated 24-well plates in triplicates. After 24, 48 or $72 \mathrm{~h}$, cells were harvested by trypsinization with TrypLE Express (Life Technologies), stained for $5 \mathrm{~min}$ with ViaCount reagent, and total numbers of viable cells were determined with flow cytometer using Cytosoft viacount module software (Guava Technologies).

Cell cycle phase analysis was performed with Guava Cell Cycle reagent using Guava Easycyte Mini Flow cytometer. miRNA mimic transfected cells were incubated in RPMI-1640 media with 10\% FBS in collagen-coated 24-well plates in triplicates. After 48 or 72 h, cells were harvested by trypsinization with TrypLE Express and slowly fixed with $10 \mathrm{ml}$ of $70 \%$ ethanol for $\geq 2 \mathrm{~h}$. After removal of ethanol by centrifuging and washing cells with PBS (-), cells were stained with Guava Cell Cycle reagent for $30 \mathrm{~min}$ and flow cytometric data were collected with flow cytometer using Cytosoft cell cycle module software (Guava Technologies). Later, flow cytometric raw data were analyzed using FCS Express 5 Pro software (DeNovo Software, Glendale, CA, USA).

Cell migration was analyzed using a wound/scratch assay. miRNA mimic transfected cells incubated in RPMI-1640 media with $10 \%$ FBS in collagen-coated 24-well plates overnight. The wound was created in cell monolayer by scratching the cells with $1 \mathrm{ml}$ micropipette tips and floating cells were removed by washing with fresh media. Real-time microscopic images were taken at $0,6,12,18$ and $24 \mathrm{~h}$ post-scratching by incubating the cells in stage-top incubator on an inverted 


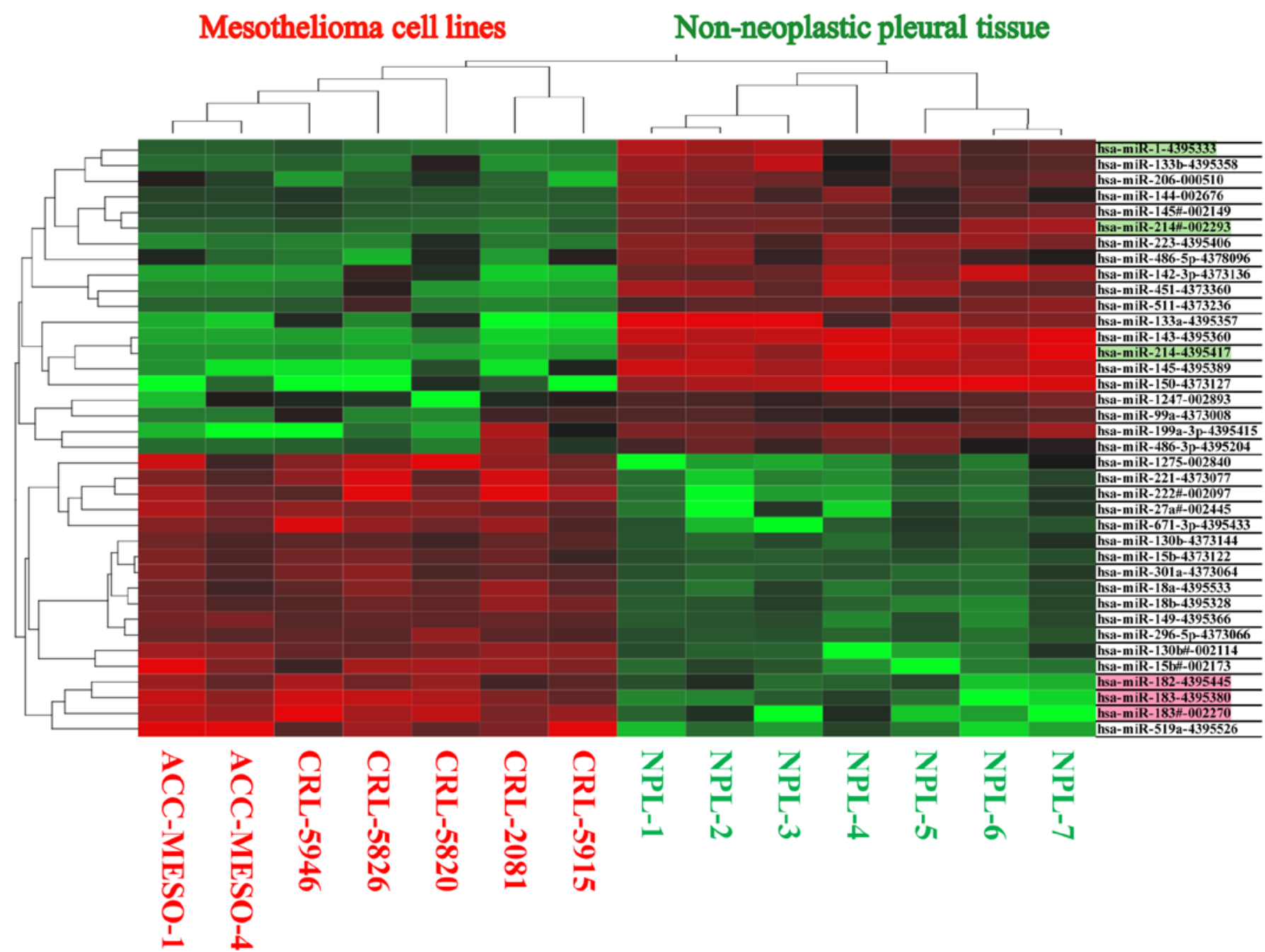

Figure 1. Supervised hierarchical clustering of differentially expressed miRNAs between mesothelioma cell lines and non-neoplastic pleural tissue. Coloring indicates miRNA expression levels from low (green) to high (red). Twenty downregulated miRNAs, including miR-1 and -214 (green underlines), show low or no expression in all seven mesothelioma cell lines, while 18 upregulated miRNAs, including miR-182 and -183 (red underlines), show increased expression.

microscope with automatic picture acquisition at given time interval (Olympus IX81 with cellSens software). The area of the wound gap that was not covered by cell migration (percentage) was determined using TScrach software (18).

Cell invasion was performed using BD FluoroBlok culture Inserts with $8-\mu \mathrm{m}$ pores (BD Biosciences, Franklin Lakes, NJ, USA) coated with matrix Matrigel (Life Technologies) according to the manufacturer's protocol. miRNA mimics transfected cells were stained with cell permeant nuclear fluorescent dye Hoechst 33342 (Life Technologies) for $10 \mathrm{~min}$ and fluorescent image (16 consecutive images with 10X objective) was captured with DP80 CCD camera using an inverted fluorescent microscope IX81. The total numbers of invading cells were calculated by analyzing fluorescent images with CellProfiler cell imaging software (19).

Cell culture in chamber-slide or cell-block preparation for immunocytochemistry. Approximately $1 \times 10^{5}$ cells were cultured in collagen coated chamber glass slide (Millicell EZ Slide: Millipore, EMD Millipore Corp., Billerica, MA, USA) overnight and fixed with $10 \%$ formalin. Each of mesothelioma cell lines was cultured for 2-3 days on 100-mm collagen-coated culture dish (Corning Life Sciences, Corning, NY, USA) and were subjected to cell-block preparation using glucomannan (HoldGel 110, EBIS1 cell-block construction kit, Asia Kizai, Tokyo, Japan) (20). Three- $\mu \mathrm{m}$-sections were prepared from formalin-fixed paraffin-embedded cell-blocks in Platinum Pro Adhesive Glass slides (Matsunami Glass, Osaka, Japan). Fully automatic immunohistochemical staining of anti-PIM1 antibody (rabbit monoclonal, Cat \#ab75776, Abcam, Cambridge, MA, USA, 1:250 dilution filled in antibody dispenser) was performed with Ventana BenchMark GX using ultraView Universal DAB Detection kit (Ventana, Roche Diagnostics, Tokyo, Japan). Similar immunohistochemical procedure was carried out with the omission of the primary antibody as a negative control.

Western blot analysis. Cell lysates were obtained from miRNA mimic transfected cells using CytoBuster Protein Extraction reagent (Novagen, Madison, WI, USA) with Halt Protease Inhibitor Cocktail (Thermo Scientific Fisher, Tokyo, Japan). Total protein $(20 \mu \mathrm{g})$ was separated on the Bolt $4-12 \%$ Bis-Tris Plus Gels (Life Technologies) with lithium dodecyl sulfate (LDS) electrophoresis at $165 \mathrm{~V}$ for $35 \mathrm{~min}$ and trans- 
Table I. Expression fold change, average CT, and average dCT value in mesothelioma cell lines (MESO) and non-neoplastic pleural tissue (NPL).

\begin{tabular}{|c|c|c|c|c|c|c|c|}
\hline \multirow[b]{2}{*}{ Assay } & \multicolumn{3}{|c|}{ Expression fold change } & \multicolumn{2}{|c|}{ Average CT } & \multicolumn{2}{|c|}{ Average $\mathrm{dCT}$} \\
\hline & MESO & NPL & P-value & MESO & NPL & MESO & NPL \\
\hline hsa-miR-519a-4395526 & 135.1 & & 0.119 & 32.5 & 39.1 & 15.1 & 21.6 \\
\hline hsa-miR-222\#-002097 & 54.9 & & 0.057 & 27.9 & 33.7 & 10.5 & 16.2 \\
\hline hsa-miR-183-4395380 & 42.6 & & 0.011 & 30.9 & 37.2 & 13.5 & 19.8 \\
\hline hsa-miR-221-4373077 & 41.7 & & 0.095 & 25.1 & 29.9 & 7.6 & 12.4 \\
\hline hsa-miR-15b\#-002173 & 31.8 & & 0.020 & 28.3 & 33.7 & 10.9 & 16.2 \\
\hline hsa-miR-1275-002840 & 21.2 & & 0.040 & 31.1 & 36.4 & 13.6 & 19.0 \\
\hline hsa-miR-183\#-002270 & 20.2 & & 0.002 & 29.4 & 36.2 & 11.9 & 18.7 \\
\hline hsa-miR-130b\#-002114 & 15.7 & & 0.018 & 31.7 & 36.3 & 14.3 & 18.8 \\
\hline hsa-miR-671-3p-4395433 & 15.0 & & 0.005 & 30.6 & 35.2 & 13.2 & 17.7 \\
\hline hsa-miR-27a\#-002445 & 10.2 & & 0.012 & 28.5 & 32.8 & 11.1 & 15.4 \\
\hline hsa-miR-149-4395366 & 8.0 & & 0.033 & 25.1 & 27.9 & 7.7 & 10.5 \\
\hline hsa-miR-18a-4395533 & 7.8 & & 0.042 & 28.1 & 30.8 & 10.6 & 13.4 \\
\hline hsa-miR-182-4395445 & 7.4 & & 0.010 & 28.6 & 32.4 & 11.2 & 14.9 \\
\hline hsa-miR-301a-4373064 & 7.1 & & 0.055 & 28.6 & 31.2 & 11.1 & 13.8 \\
\hline hsa-miR-18b-4395328 & 7.0 & & 0.011 & 32.2 & 35.1 & 14.7 & 17.7 \\
\hline hsa-miR-15b-4373122 & 6.3 & & 0.026 & 25.9 & 28.2 & 8.4 & 10.7 \\
\hline hsa-miR-296-5p-4373066 & 6.1 & & 0.021 & 31.0 & 33.4 & 13.5 & 15.9 \\
\hline hsa-miR-130b-4373144 & 3.6 & & 0.014 & 29.6 & 31.3 & 12.1 & 13.9 \\
\hline hsa-miR-99a-4373008 & 3.4 & & 0.249 & 26.3 & 26.0 & 8.8 & 8.5 \\
\hline hsa-miR-486-3p-4395204 & & 26.3 & 0.016 & 38.0 & 29.4 & 20.6 & 11.9 \\
\hline hsa-miR-199a-3p-4395415 & & 41.2 & 0.000 & 35.5 & 23.5 & 18.0 & 6.1 \\
\hline hsa-miR-511-4373236 & & 134.6 & 0.002 & 39.1 & 28.9 & 21.6 & 11.4 \\
\hline hsa-miR-1247-002893 & & 171.0 & 0.001 & 34.2 & 25.1 & 16.8 & 7.7 \\
\hline hsa-miR-142-3p-4373136 & & 352.0 & 0.000 & 38.2 & 25.8 & 20.7 & 8.3 \\
\hline hsa-miR-206-000510 & & 490.7 & 0.020 & 37.1 & 26.8 & 19.6 & 9.4 \\
\hline hsa-miR-486-5p-4378096 & & 588.6 & 0.038 & 35.8 & 25.9 & 18.3 & 8.5 \\
\hline hsa-miR-451-4373360 & & 759.8 & 0.009 & 39.1 & 26.6 & 21.6 & 9.2 \\
\hline hsa-miR-144-002676 & & $1,223.0$ & 0.040 & 40.0 & 30.4 & 22.6 & 12.9 \\
\hline hsa-miR-145\#-002149 & & $1,340.9$ & 0.014 & 40.0 & 29.9 & 22.6 & 12.5 \\
\hline hsa-miR-133b-4395358 & & $1,643.8$ & 0.112 & 39.2 & 28.1 & 21.7 & 10.7 \\
\hline hsa-miR-214\#-002293 & & $2,788.9$ & 0.005 & 39.9 & 28.7 & 22.4 & 11.3 \\
\hline hsa-miR-223-4395406 & & $2,977.7$ & 0.001 & 32.7 & 20.6 & 15.2 & 3.1 \\
\hline hsa-miR-1-4395333 & & $6,705.6$ & 0.049 & 40.0 & 28.3 & 22.6 & 10.8 \\
\hline hsa-miR-150-4373127 & & $10,404.8$ & 0.001 & 37.3 & 21.7 & 19.9 & 4.2 \\
\hline hsa-miR-145-4395389 & & $12,025.2$ & 0.004 & 37.7 & 22.6 & 20.2 & 5.2 \\
\hline hsa-miR-133a-4395357 & & $13,032.1$ & 0.050 & 37.9 & 23.6 & 20.4 & 6.2 \\
\hline hsa-miR-214-4395417 & & $32,285.8$ & 0.001 & 40.0 & 25.0 & 22.5 & 7.5 \\
\hline hsa-miR-143-4395360 & & $55,283.3$ & 0.002 & 39.7 & 24.0 & 22.3 & 6.5 \\
\hline
\end{tabular}

ferred onto a polyvinylidene difluoride (PVDF) membrane using iBlot transfer stack (Life Technologies) at $20 \mathrm{~V}$ for $7 \mathrm{~min}$. The protein-transferred membrane was processed on BenchPro 4100 Western System (Life Technologies) with primary antibodies, anti-PIM1 antibody (1:500, rabbit monoclonal, ab75776, Abcam) and anti-GAPDH antibody (1:500, rabbit polyclonal sc-25778, Santa Cruz Biotechnology, Dallas, TX, USA) and biotin labeled secondary antibody [Biotin-XX goat anti-rabbit $\operatorname{IgG}(\mathrm{H}+\mathrm{L})$ (Life Technologies)]. The membrane was washed and stained with Qdot 625 streptavidin conjugate (Life Technologies) for $30 \mathrm{~min}$ and bands were detected using E-Gel Imager (Life Technologies). 


\section{ACC-MESO1}
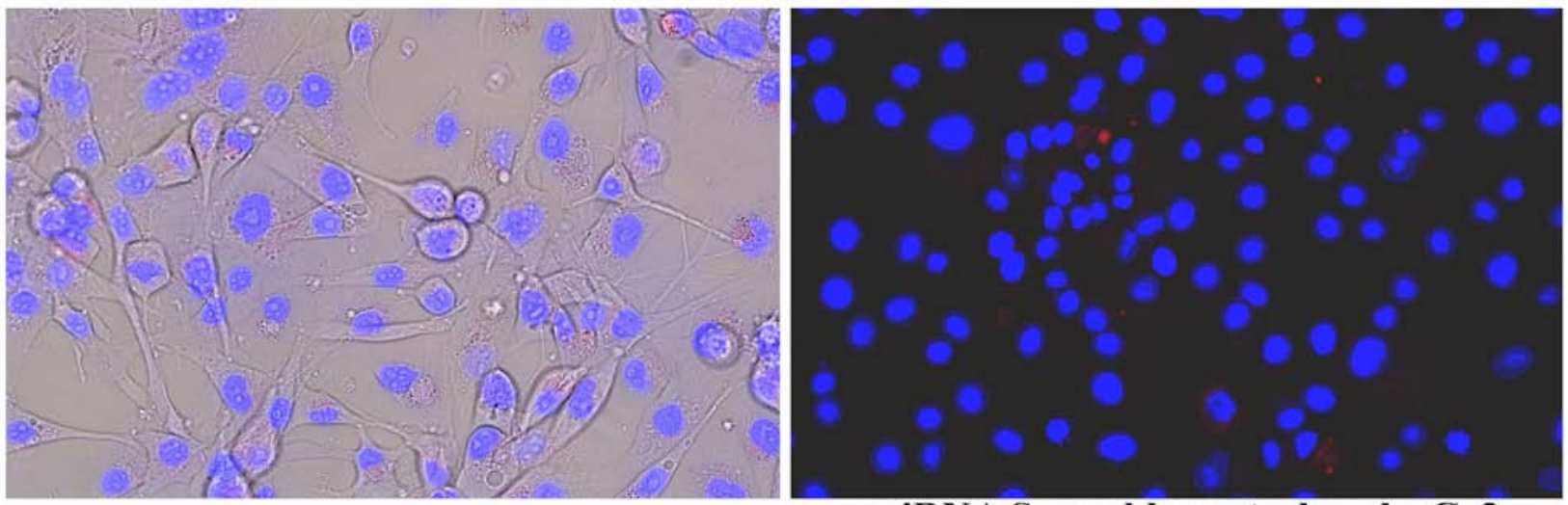

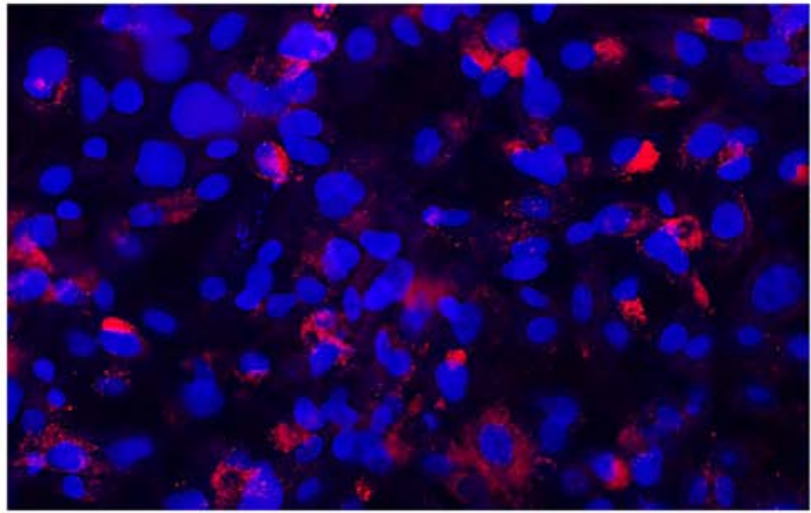

miR-182 SmartFlare probe Cy3
miRNA Scramble control probe $\mathrm{Cy3}$

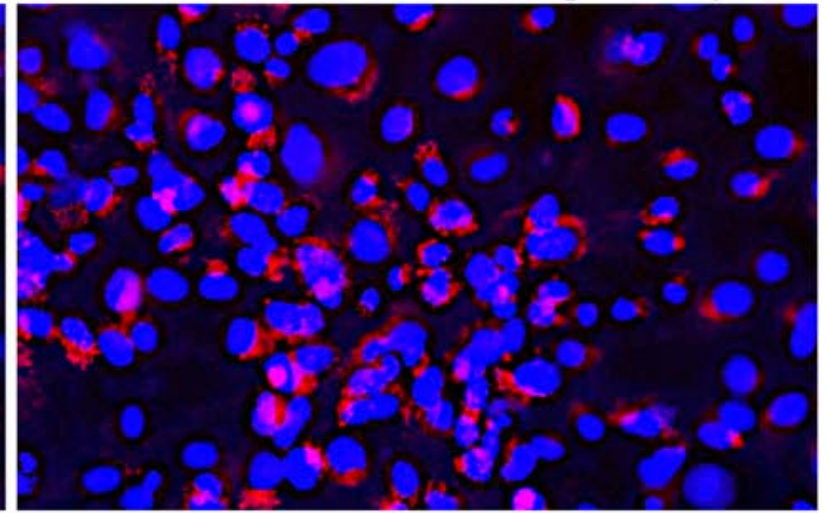

miR-183 SmartFlare probe Cy3

Figure 2. Validation of miRNA expression in mesothelioma cell lines. Using SmartFlare miRNA probe, mesothelioma cell line, ACC-Meso-1 show expression of miR-182 (lower left) and miR-183 (lower right) in cytoplasm. Scrambled miRNA probe, utilized as the negative control, show no fluorescence in the cytoplasm of mesothelioma cell line.

Statistical analysis. The TaqMan Low Density microRNA Array data were analyzed with DataAssist software and JMP 9. All other statistical analysis was performed with JMP 9 software (SAS Institute, Inc., Cary, NC, USA).

\section{Results}

Differential miRNA expression in mesothelioma cells and its validation. Out of 754 analyzable miRNAs by TaqMan Low Density Array Human miRNA Cards, significant number of miRNA were found to be differentially expressed between mesothelioma cell lines (CRL-2081, -5820, -5826, -5915, -5946, ACC-Meso-1, ACC-Meso-4) and non-neoplastic mesothelial tissue. We found increased expression of 18 miRNAs and reduced or absent expression of 20 miRNAs in mesothelioma cell lines (Fig. 1 and Table I). Using SmartFlare miRNA probes and scrambled negative control probe, we found all of these 7 mesothelioma cell lines have increased miR-182, miR-183 (Fig. 2). We also validated miRNA expression in these mesothelioma cell lines by individual microRNA expression assay (data not shown).

miR-1 and miR-214 mimic transfection inhibits cell growth, cell cycle progression, migration and invasion of mesothelioma cells. Two mesothelioma cell lines, ACC-Meso-1 and
CRL-5915 were transfected with hsa-miR-1, and hsa-miR-214 miRNA mimics, and negative control miRNA mimic. Cell proliferation was significantly suppressed in mesothelioma cells transfected with miR-1 and miR-214 mimic compared to negative control group at 48 and $72 \mathrm{~h}$ after transfection by flow cytometric analysis of viable cells $(\mathrm{p}<0.05$, Fig. $3 \mathrm{~A}$ ). Furthermore, flow cytometric analysis indicated that proportion of cells in G0/G1 phase was increased significantly, whereas cells in $\mathrm{S}$ and $\mathrm{G} 2 / \mathrm{M}$ phase was significantly decreased in miR-1 and miR-214 mimic transfected cells compared to control group ( $\mathrm{p}<0.05$, Fig. $3 \mathrm{~B}$ ). The cell migration analysis by wound scratch assay showed decreased speed of cell migration with hsa-miR-1 and hsa-miR-214 miRNA mimics compared to negative control miRNA mimic (Fig. 3C). Matrigel invasion chamber assay showed less number of cell invasion through the Matrigel in cells transfected with hsa-miR-1 and hsa-miR-214 mimics ( $\mathrm{p}<0.05$, Fig. 3D).

Target prediction database of miR-1 and miR-214 shows PIMI as possible target in mesothelioma. To analyze the underlying mechanism of miR-1 and miR-214 in mesothelioma cell carcinogenesis, online miRTarBase (http://mirtarbase.mbc.nctu. edu.tw/php/search.php) was used to search for potential targets of miR-1 and miR-214, and we noted PIM1 was the target gene of both miR-1, miR-214. We also found similar result 


\section{A. Cell proliferation analysis}

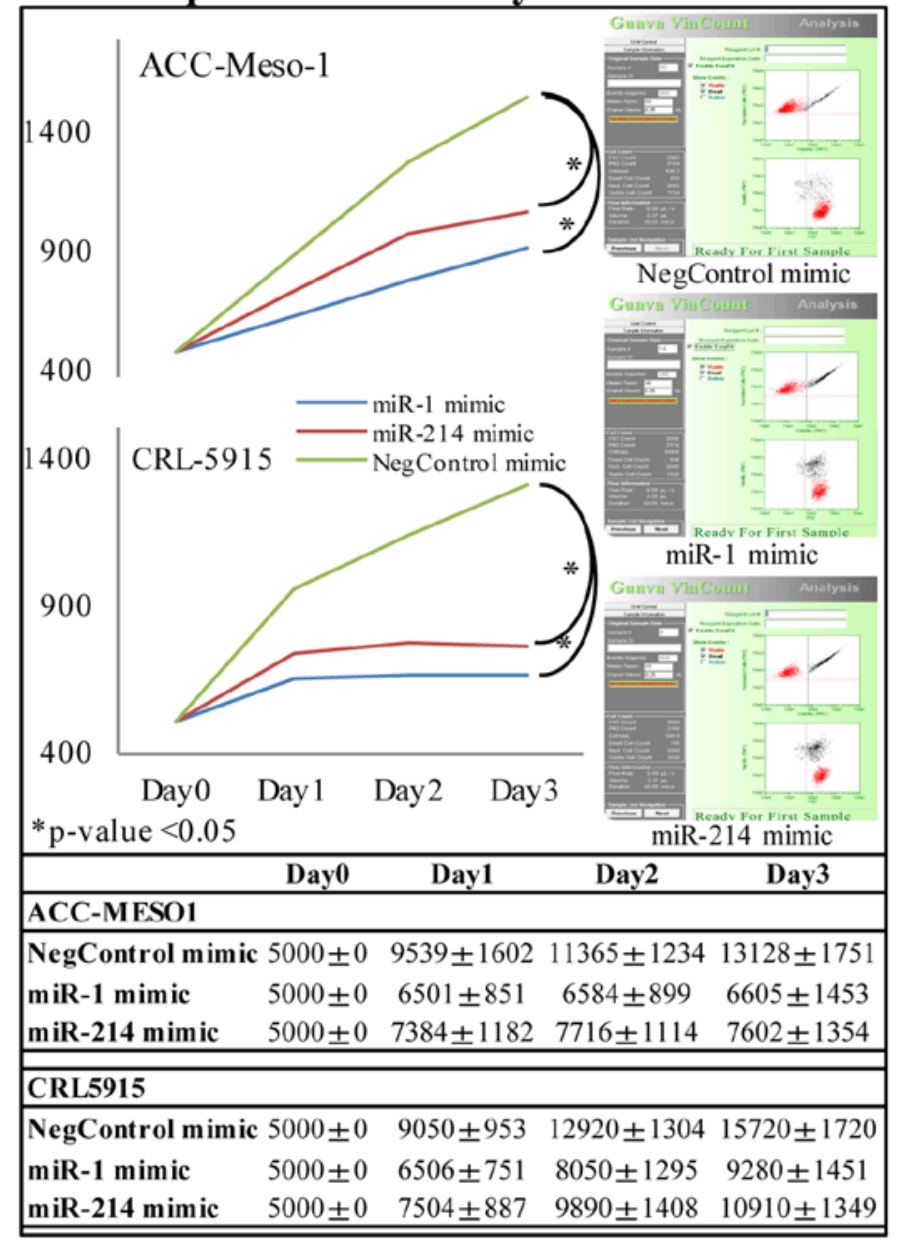

C. Invasion assay

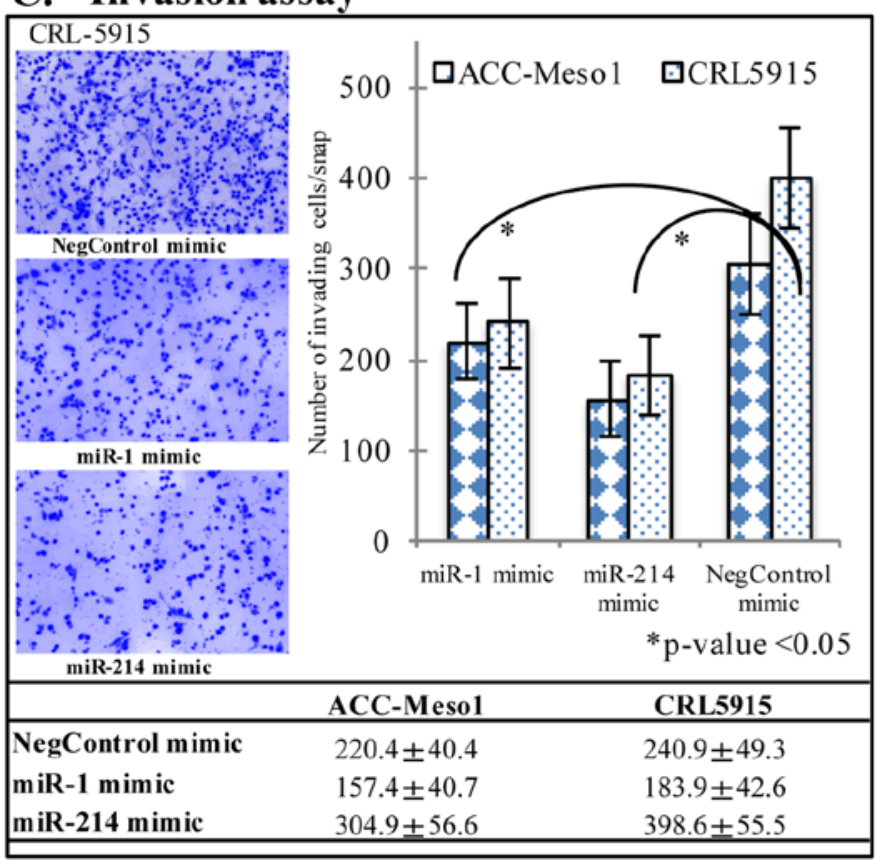

B. Cell cycle analysis

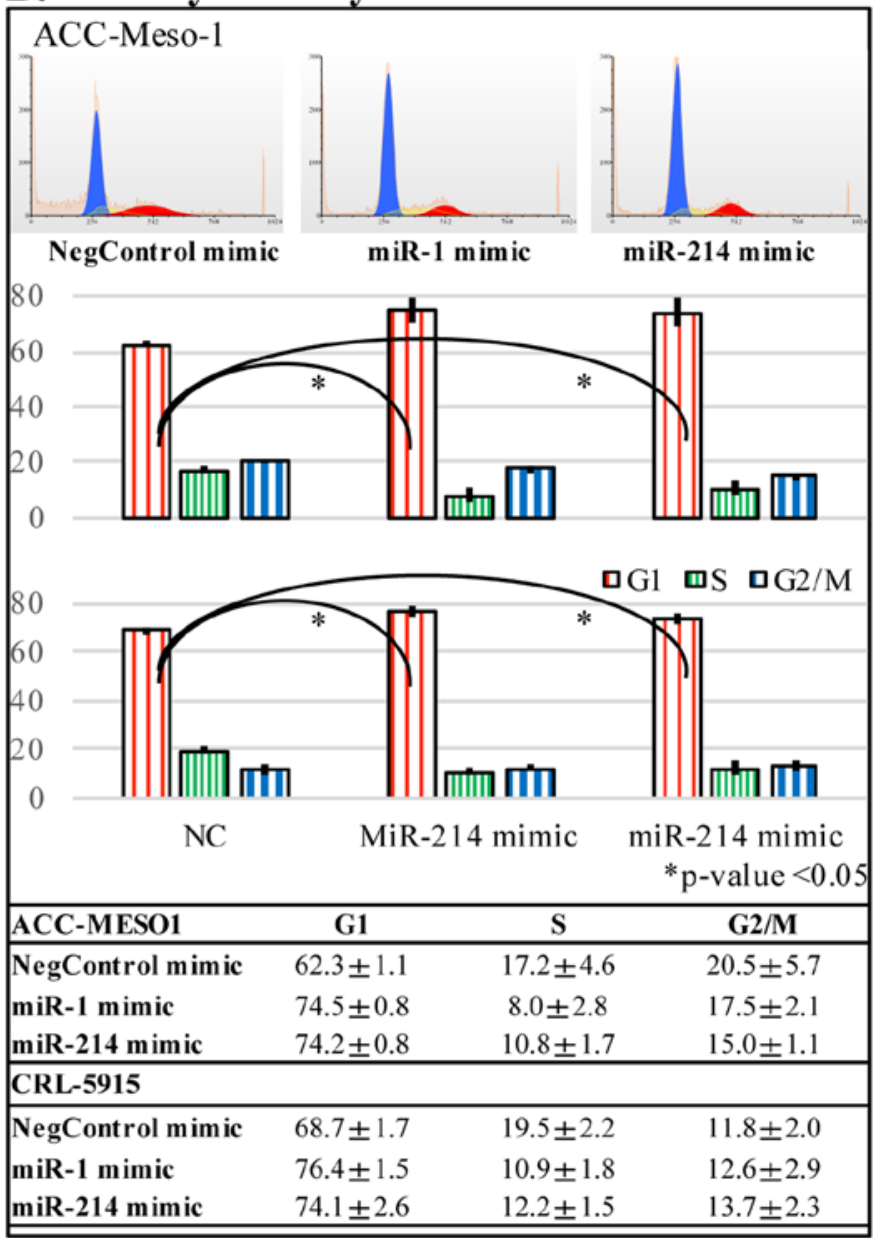

D. Migration assay

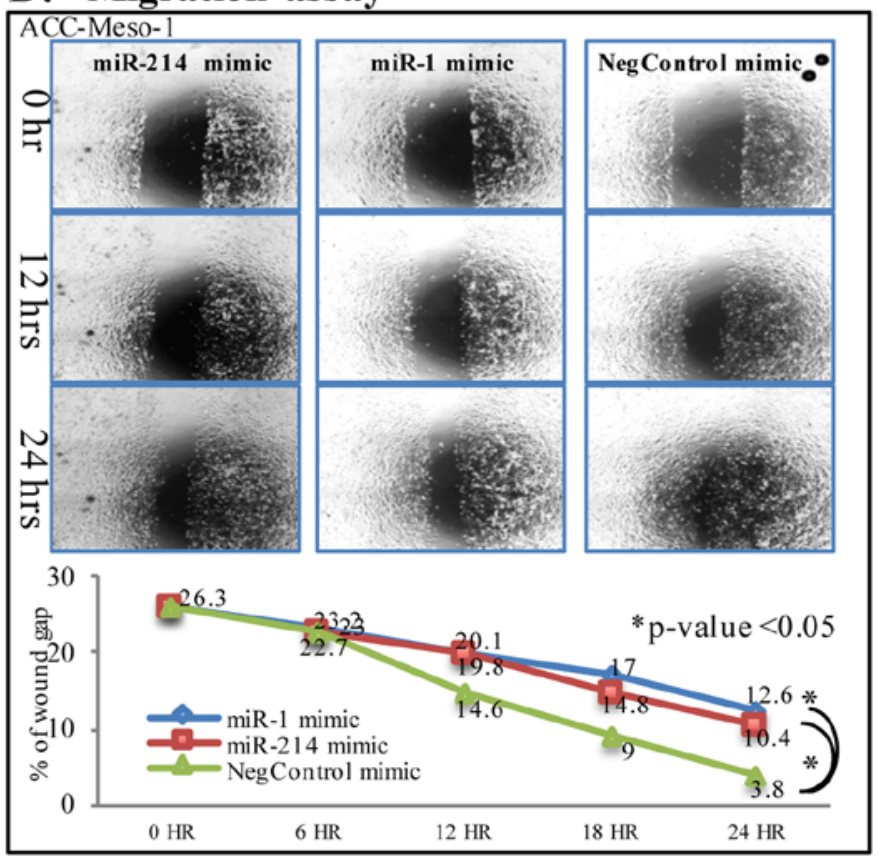

Figure 3. (A) Cell proliferation analysis. Transfection of mesothelioma cell lines, ACC-Meso-1 and CRL5915, with miR-1 and miR-214 mimic for one, two or three days shows the suppression of proliferation of viable cells in increasing order compared to negative control miRNA mimic (data acquired by flow cytometric analysis using Guava viaCount reagent, p<0.05). (B) Cell cycle assay. Cell cycle phase analysis was performed with Guava Cell Cycle reagent using Guava Easycyte Mini Flow cytometer. miR-1 and miR-214 mimic transfected mesothelioma cells show increased G1 phase compared to negative control miRNA mimic, suggesting G1 arrest of mesothelioma cell line (data acquired by flow cytometric analysis using Guava cell cycle reagent, p<0.05). (C) Invasion assay. Transfection of mesothelioma cell lines, ACC-Meso-1 and CRL5915, with miR-1 and miR-214 mimic for two or three days reduce invasiveness of mesothelioma cells through Matrigel compared to negative control miRNA mimic ( $<<0.05)$. Left panel figures inverted from the fluorescent images using CellProfiler Image analysis software. (D) Migration assay. Mesothelioma cells, ACC-Meso-1 showed slow migration of cells transfected with miR-1 and miR-214 mimic transfection compared to that in negative control miRNA mimic transfection $(\mathrm{p}<0.05)$. 

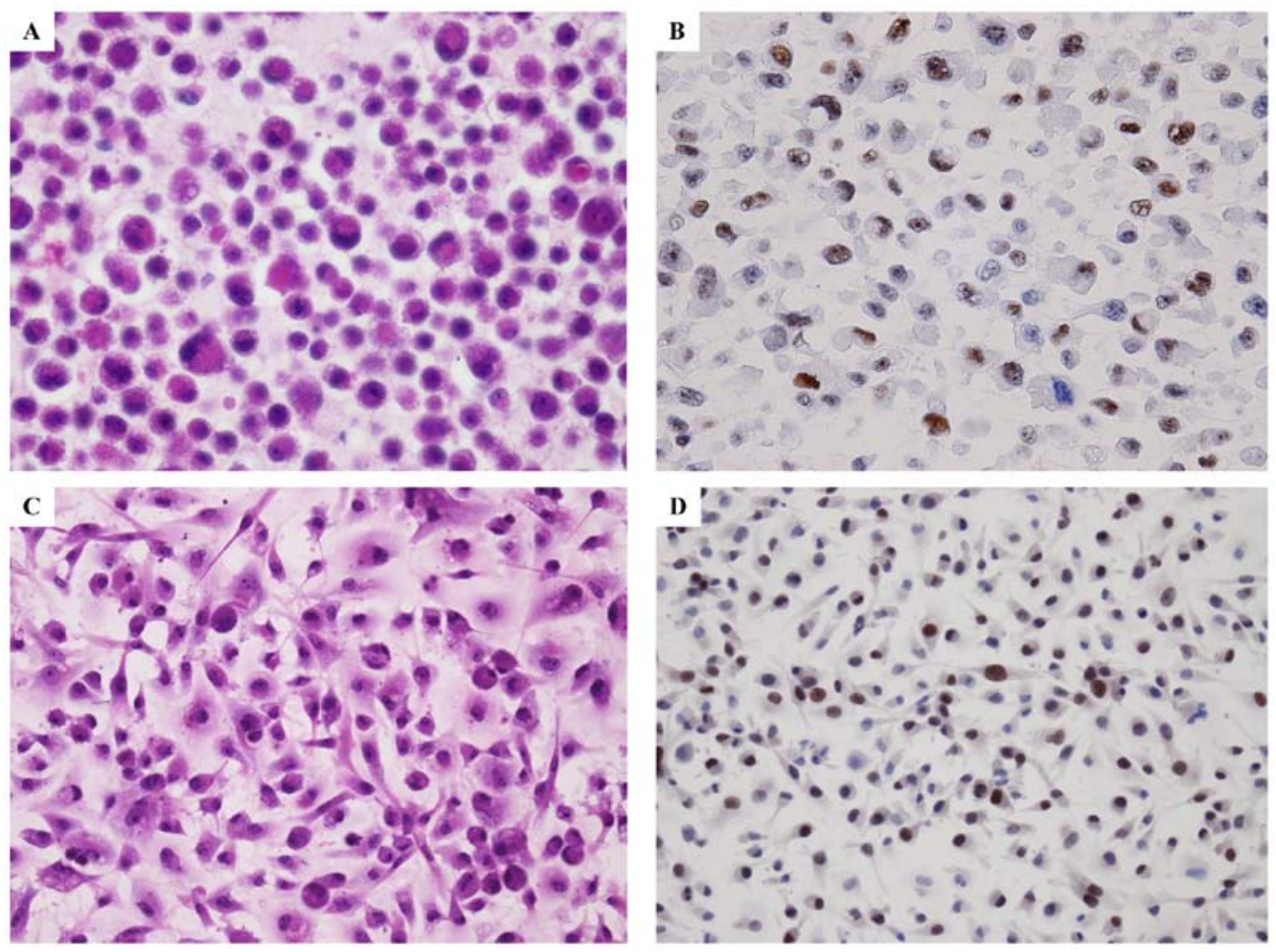

Figure 4. Immunohistochemical expression of PIM1 in mesothelioma cell lines. Nuclear expression of PIM1 is present in mesothelioma cell lines, ACCMeso-1 [A and B) cell-block preparation] and CRL5915 [(C and D) chamber-slide preparation]. (A and C) Hematoxylin and eosin staining x200; (B and D) Anti-PIM1 immunohistochemical staining x200.

ACC-MESO1
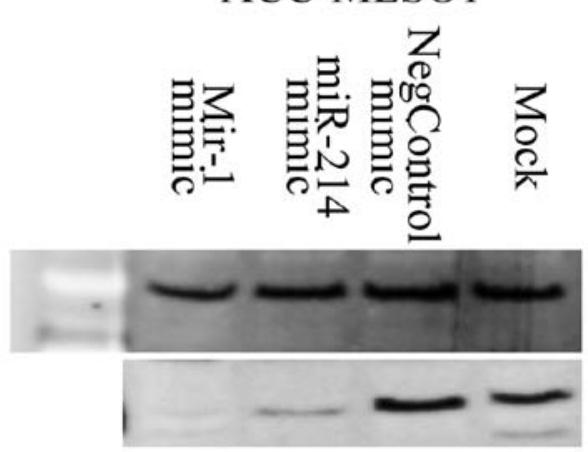

CRL-5915
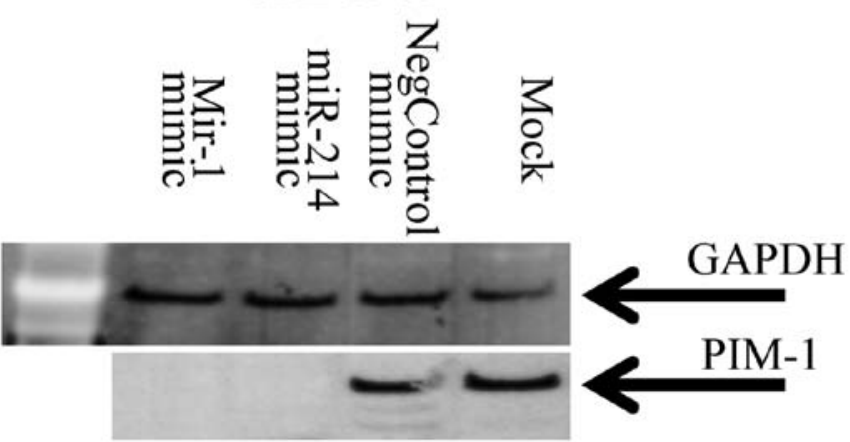

Figure 5. Western blot analysis of PIM-1 expression. Soluble proteins ( $30 \mu \mathrm{g})$ were analyzed by western blot analysis for detection of PIM1 using anti-PIM1 antibody. Anti-GAPDH antibody was used as the housekeeping gene control. ACC-Meso-1 show reduced expression of PIM1 protein compared to negative control miRNA mimic and mock.

from TargetScan (www.targetscan.org) and miRanda (www. microrna.org). Thus, we investigated whether the PIM1 was expressed in mesothelioma cell lines or not. By immunocytochemical study of cell-blocks prepared from mesothelioma cell lines, we found PIM1 was highly expressed in all of the seven mesothelioma cells lines (Fig. 4B). In addition, we also investigated the expression of PIM1 in mesothelioma cell line cultured directly in chamber-slide (Fig. 4D).

Transfection of miR-1 and miR-214 downregulates PIM1 in mesothelioma cell lines. To determine if miR-1 and miR-214 target PIM1 in mesothelioma, the miR-1, miR-214 mimic or a control were transfected into ACC-Meso-1 and CRL5915 cells.
Both of the cell lines used in this study, showed PIM1 expression. The serum starved cells transfected with miR-1 and miR-214 mimic showed reduced PIM1 protein, respectively, after 2 days and 3 days of transfection compared to control miRNA mimic (Fig. 5). These results confirmed that miR-1 and miR-214 mimic transfection downregulated PIM1 expression in mesothelioma cells.

\section{Discussion}

Malignant pleural mesothelioma is an aggressive cancer as current treatment modalities do not improve patient survival (21). Present understanding of molecular mechanism is not 
yet sufficient to improve the management of these patients. miRNAs for their powerful regulatory potential are recently considered as potential therapeutic targets to various human malignancies, including malignant mesothelioma miR-31 (11), $\mathrm{miR}-34 \mathrm{~b} / \mathrm{c}$ (12). In addition, the investigation of the target genes involved in various malignancies are also possible by analyzing aberration of the miRNA expression. Many reports, summarized by Truini et al (10), have identified various miRNAs associated with mesothelioma. Here we add miR-1, miR-214 and its prediction target PIM1 gene as possible target of therapeutic potential in mesothelioma.

Previous reports have shown downregulation of miR-1 and mir-214 in various human malignancies. miR-1 expression is reported to be downregulated in colon cancer (22), gastric cancer (23), lung cancer (24), prostate cancer (25), renal cancer (26), and esophageal cancer (27). Xu et al (28) have shown low miR-1 expression in mesothelioma and transfection of precursor miR-1 been shown to suppress cell growth and induce apoptosis. In our study, we found negligible miR-1 in mesothelioma cell line by real-time RT PCR method and transaction of miR-1 mimic showed reduced cell proliferation with cell cycle G1 arrest, reduced cell migration and invasive nature by in vitro experiments. Aberrant expression of miR-214 has been reported in the past with its downregulation in liver cancers $(29,30)$, cervical cancer $(31)$, prostate cancer (32), and upregulation in lung cancer (33), bladder cancer (34) and esophageal cancer (35). In malignant mesothelioma, we found no expression of miR-1 and miR-214 in mesothelioma cell lines.

The loss of expression of miR-1 and miR-214 may have potential as the diagnostic biomarker of malignant mesothelioma. The transfection of miR-1 or miR-214 both inhibits cell proliferation, cell migration and invasion. The histopathological differentiation of early malignant mesothelioma from reactive mesothelial proliferation has been performed even in an immunohistochemical study (36). The assessment of miR-1 and miR-214 expression may be utilized as differentiation marker of mesothelioma from reactive mesothelial proliferation. In situ hybridization with miR-1 and/or miR-214 probes could be utilized to differentiate mesothelioma from reactive mesothelial tissue. However, further analysis of the miRNA expression in human mesothelioma and reactive mesothelial hyperplasia tissue is necessary. We intend to perform such analysis using human tissue in future.

By target prediction analysis using online miRTarBase release 6.1 (37), we analyzed the common target of miR-1 and miR-214 and we primarily focused on PIM1 proto-oncogene. We analyzed whether the mesothelioma cell line express PIM1 or not. We investigated PIM1 expression by immunohistochemistry of cell lines cultured directly on collagen coated chamber-slide and cell-block. We found that PIM1 was highly expressed in all seven mesothelioma cell lines that we analyzed in our miRNA profiling study. In addition, by transfecting miR-1 and miR-214 mimic to two cell lines, ACC-MESO-1, and CRL-5915, we also found the downregulation of PIM1 protein expression suggesting the role of miR-1, and miR-214 in inhibition of PIM1 expression. The expression of PIM1 gene in human cancer has been studied in great depth and was found to be overexpressed in a wide range of human tumors. PIM expression level has shown favorable prognosis in prostate cancer (38), pancreatic ductal carcinoma (39), and non-small cell lung cancer (40), and unfavorable prognosis in gastric cancer (41), and squamous cell carcinoma of the head and neck (42). As yet, there is no published report on PIM1 expression in malignant mesothelioma. This is the first report on increased PIM1 expression in mesothelioma. Further, novel molecules inhibiting PIM kinases have been evaluated in preclinical studies, demonstrating to be effective and with a favorable toxicity profile. Given the promising results, some of these compounds are currently under investigation in clinical trials (43). However, its application and therapeutic potential in mesothelioma needs additional investigation to assess the significance in mesothelioma.

In conclusion, we have shown that loss of miR-1 and miR-214 expression and high expression of their target gene, PIM1, in malignant mesothelioma. By miRNA mimic transfection study we consider the loss of miR-1 and miR-214 play a role in cell proliferation, invasion, and migration in mesothelioma, probably by downregulation of PIM1. It remains feasible that loss of miR-1 and miR-214 expression may behave as an oncogene in mesothelioma and have potential as an oncogenic target. miR-1 and miR-214 may be attractive targets for future mesothelioma therapeutic studies.

\section{Acknowledgements}

Part of this study was carried out at the Analysis Center of Life Science, Hiroshima University.

\section{References}

1. Weill H, Hughes JM and Churg AM: Changing trends in US mesothelioma incidence. Occup Environ Med 61: 438-441, 2004.

2. Centers for Disease Control and Prevention (CDC): Malignant mesothelioma mortality - United States, 1999-2005. MMWR Morb Mortal Wkly Rep 58: 393-396, 2009.

3. Takeshima Y, Inai K, Amatya VJ, Gemba K, Aoe K, Fujimoto N, Kato $\mathrm{K}$ and Kishimoto T: Accuracy of pathological diagnosis of mesothelioma cases in Japan: Clinicopathological analysis of 382 cases. Lung Cancer 66: 191-197, 2009.

4. Amatya VJ, Takeshima Y, Aoe K, Fujimoto N, Okamoto T, Yamada T, Kishimoto T, Morimoto C and Inai K: CD9 expression as a favorable prognostic marker for patients with malignant mesothelioma. Oncol Rep 29: 21-28, 2013.

5. Pass HI, Vogelzang N, Hahn S and Carbone M: Malignant pleural mesothelioma. Curr Probl Cancer 28: 93-174, 2004.

6. Robinson BW and Lake RA: Advances in malignant mesothelioma. N Engl J Med 353: 1591-1603, 2005.

7. Montanaro F, Rosato R, Gangemi M, Roberti S, Ricceri F, Merler E, Gennaro V, Romanelli A, Chellini E, Pascucci C, et al: Survival of pleural malignant mesothelioma in Italy: A population-based study. Int J Cancer 124: 201-207, 2009.

8. He L and Hannon GJ: MicroRNAs: Small RNAs with a big role in gene regulation. Nat Rev Genet 5: 522-531, 2004.

9. Drakaki A and Iliopoulos D: MicroRNA gene networks in Oncogenesis. Curr Genomics 10: 35-41, 2009.

10. Truini A, Coco S, Alama A, Genova C, Sini C, Dal Bello MG, Barletta G, Rijavec E, Burrafato G, Boccardo F, et al: Role of microRNAs in malignant mesothelioma. Cell Mol Life Sci 71: 2865-2878, 2014.

11. Busacca S, Germano S, De Cecco L, Rinaldi M, Comoglio F, Favero F, Murer B, Mutti L, Pierotti M and Gaudino G: MicroRNA signature of malignant mesothelioma with potential diagnostic and prognostic implications. Am J Respir Cell Mol Biol 42: 312-319, 2010.

12. Kubo T, Toyooka S, Tsukuda K, Sakaguchi M, Fukazawa T, Soh J, Asano H, Ueno T, Muraoka T, Yamamoto H, et al: Epigenetic silencing of microRNA-34b/c plays an important role in the pathogenesis of malignant pleural mesothelioma. Clin Cancer Res 17: 4965-4974, 2011. 
13. Pass HI, Goparaju C, Ivanov S, Donington J, Carbone M, Hoshen M, Cohen D, Chajut A, Rosenwald S, Dan H, et al: hsa-miR-29c* is linked to the prognosis of malignant pleural mesothelioma. Cancer Res 70: 1916-1924, 2010.

14. Gee GV, Koestler DC, Christensen BC, Sugarbaker DJ, Ugolini D, Ivaldi GP, Resnick MB, Houseman EA, Kelsey KT and Marsit CJ: Downregulated microRNAs in the differentia diagnosis of malignant pleural mesothelioma. Int J Cancer 127: $2859-2869,2010$

15. Santarelli L, Strafella E, Staffolani S, Amati M, Emanuelli M, Sartini D, Pozzi V, Carbonari D, Bracci M, Pignotti E, et al: Association of miR-126 with soluble mesothelin-related peptides, a marker for malignant mesothelioma. PLoS One 6: e18232, 2011.

16. Kirschner MB, Cheng YY, Badrian B, Kao SC, Creaney J, Edelman JJ, Armstrong NJ, Vallely MP, Musk AW, Robinson BW, et al: Increased circulating miR-625-3p: A potential biomarker for patients with malignant pleural mesothelioma. J Thorac Oncol 7: 1184-1191, 2012

17. Usami N, Fukui T, Kondo M, Taniguchi T, Yokoyama T, Mori S, Yokoi K, Horio Y, Shimokata K, Sekido Y, et al: Establishment and characterization of four malignant pleural mesothelioma cel lines from Japanese patients. Cancer Sci 97: 387-394, 2006.

18. Gebäck T, Schulz MM, Koumoutsakos P and Detmar M TScratch: A novel and simple software tool for automated analysis of monolayer wound healing assays. Biotechniques 46 : 265-274, 2009

19. Lamprecht MR, Sabatini DM and Carpenter AE: CellProfiler: Free, versatile software for automated biological image analysis. Biotechniques 42: 71-75, 2007.

20. Amatya VJ, Takeshima Y, Kushitani K, Yamada T, Morimoto C and Inai K: Overexpression of CD26/DPPIV in mesothelioma tissue and mesothelioma cell lines. Oncol Rep 26: 1369-1375, 2011.

21. Milano MT and Zhang H: Malignant pleural mesothelioma: A population-based study of survival. J Thorac Oncol 5: 1841-1848, 2010.

22. Furukawa S, Kawasaki Y, Miyamoto M, Hiyoshi M, Kitayama J and Akiyama T: The miR-1-NOTCH3-Asef pathway is important for colorectal tumor cell migration. PLoS One 8: e80609, 2013

23. Han C, Zhou Y, An Q, Li F, Li D, Zhang X, Yu Z, Zheng L, Duan Z and Kan Q: MicroRNA-1 (miR-1) inhibits gastric cancer cell proliferation and migration by targeting MET. Tumour Biol 36: 6715-6723, 2015.

24. Nasser MW, Datta J, Nuovo G, Kutay H, Motiwala T, Majumder S, Wang B, Suster S, Jacob ST and Ghoshal K: Down-regulation of micro-RNA-1 (miR-1) in lung cancer. Suppression of tumorigenic property of lung cancer cells and their sensitization to doxorubicin-induced apoptosis by miR-1. J Biol Chem 283: 33394-33405, 2008

25. Karatas OF, Guzel E, Suer I, Ekici ID, Caskurlu T, Creighton CJ, Ittmann $M$ and Ozen M: miR-1 and miR-133b are differentially expressed in patients with recurrent prostate cancer. PLoS One 9: e98675, 2014

26. Kawakami K, Enokida H, Chiyomaru T, Tatarano S, Yoshino H, Kagara I, Gotanda T, Tachiwada T, Nishiyama K, Nohata N, et al The functional significance of miR-1 and miR-133a in renal cell carcinoma. Eur J Cancer 48: 827-836, 2012.

27. Du YY, Zhao LM, Chen L, Sang MX, Li J, Ma M and Liu JF: The tumor-suppressive function of miR-1 by targeting LASP1 and TAGLN2 in esophageal squamous cell carcinoma. J Gastroenterol Hepatol: Sep 28, 2015 (Epub ahead of print). doi: 10.1111/jgh.13180.

28. Xu Y, Zheng M, Merritt RE, Shrager JB, Wakelee H, Kratzke RA and Hoang CD: miR-1 induces growth arrest and apoptosis in malignant mesothelioma. Chest 144: 1632-1643, 2013.
29. Xia H, Ooi LL and Hui KM: miR-214 targets $\beta$-catenin pathway to suppress invasion, stem-like traits and recurrence of human hepatocellular carcinoma. PLoS One 7: e44206, 2012.

30. Li B, Han Q, Zhu Y, Yu Y, Wang J and Jiang X: Down-regulation of miR-214 contributes to intrahepatic cholangiocarcinoma metastasis by targeting Twist. FEBS J 279: 2393-2398, 2012.

31. Wang F, Liu M, Li X and Tang H: miR-214 reduces cell survival and enhances cisplatin-induced cytotoxicity via down-regulation of Bcl2l2 in cervical cancer cells. FEBS Lett 587: 488-495, 2013.

32. Srivastava A, Goldberger H, Dimtchev A, Ramalinga M, Chijioke J, Marian C, Oermann EK, Uhm S, Kim JS, Chen LN, et al: MicroRNA profiling in prostate cancer - the diagnostic potential of urinary miR-205 and miR-214. PLoS One 8: e76994, 2013.

33. Long H, Wang Z, Chen J, Xiang T, Li Q, Diao X and Zhu B: microRNA-214 promotes epithelial-mesenchymal transition and metastasis in lung adenocarcinoma by targeting the suppressorof-fused protein (Sufu). Oncotarget 6: 38705-38718, 2015.

34. Wang J, Zhang X, Wang L, Yang Y, Dong Z, Wang H, Du L and Wang C: MicroRNA-214 suppresses oncogenesis and exerts impact on prognosis by targeting PDRG1 in bladder cancer. PLoS One 10: e0118086, 2015.

35. Phatak P, Byrnes KA, Mansour D, Liu L, Cao S, Li R, Rao JN, Turner DJ, Wang JY and Donahue JM: Overexpression of miR-214-3p in esophageal squamous cancer cells enhances sensitivity to cisplatin by targeting survivin directly and indirectly through CUG-BP1. Oncogene: doi: 10.1038/onc.2015.271. [Epub ahead of print].

36. Tsukiji H, Takeshima Y, Amatya VJ, Kushitani K and Inai K: Myogenic antigen expression is useful for differentiation between epithelioid mesothelioma and non-neoplastic mesothelial cells. Histopathology 56: 969-974, 2010.

37. Hsu SD, Tseng YT, Shrestha S, Lin YL, Khaleel A, Chou CH, Chu CF, Huang HY, Lin CM, Ho SY, et al: miRTarBase update 2014: An information resource for experimentally validated miRNA-target interactions. Nucleic Acids Res 42D: D78-D85, 2014.

38. Dhanasekaran SM, Barrette TR, Ghosh D, Shah R, Varambally S, Kurachi K, Pienta KJ, Rubin MA and Chinnaiyan AM: Delineation of prognostic biomarkers in prostate cancer. Nature 412: 822-826, 2001.

39. Reiser-Erkan C, Erkan M, Pan Z, Bekasi S, Giese NA, Streit S, Michalski CW, Friess $\mathrm{H}$ and Kleeff J: Hypoxia-inducible protooncogene Pim-1 is a prognostic marker in pancreatic ductal adenocarcinoma. Cancer Biol Ther 7: 1352-1359, 2008.

40. Warnecke-Eberz U, Bollschweiler E, Drebber U, Pohl A, Baldus SE, Hoelscher AH and Metzger R: Frequent downregulation of pim-1 mRNA expression in non-small cell lung cancer is associated with lymph node metastases. Oncol Rep 20: 619-624, 2008.

41. Warnecke-Eberz U, Bollschweiler E, Drebber U, Metzger R, Baldus SE, Hölscher AH and Mönig S: Prognostic impact of protein overexpression of the proto-oncogene PIM-1 in gastric cancer. Anticancer Res 29: 4451-4455, 2009.

42. Peltola K, Hollmen M, Maula SM, Rainio E, Ristamäki R, Luukkaa M, Sandholm J, Sundvall M, Elenius K, Koskinen PJ, et al: Pim-1 kinase expression predicts radiation response in squamocellular carcinoma of head and neck and is under the control of epidermal growth factor receptor. Neoplasia 11: 629-636, 2009

43. Mondello P, Cuzzocrea S and Mian M: Pim kinases in hematological malignancies: Where are we now and where are we going? J Hematol Oncol 7: 95, 2014. 